\title{
A NOTE ON THE ISOPERIMETRIC INEQUALITY AND ITS STABILITY
}

\author{
XIANG GAO
}

Abstract. In this paper, we deals with isoperimetric-type inequalities for closed convex curves in the Euclidean plane $\mathbb{R}^{2}$. We derive a family of parametric inequalities involving the following geometric functionals associated to a given convex curve with a simple Fourier series proof: length, area of the region included by the curve, area of the domain enclosed by the locus of curvature centers and integral of the radius of curvature. By using our isoperimetric-type inequalities, we also obtain some new geometric Bonnesen-type inequalities. Furthermore we investigate stability properties of such inequalities (near equality implies curve nearly circular).

Mathematics subject classification (2010): Primary 52A38, Secondary 52A40.

Keywords and phrases: Isoperimetric inequality, Fourier series, stability.

\section{REFERENCES}

[1] S. L. PAN, H. Zhang, A reverse isoperimetric inequality for convex plane curves, Beitrage Algebra Geom. 48 (2007), 303-308.

[2] S. L. PAN, J. N. YANG, On a non-local perimeter-preserving curve evolution problem for convex plane curves, Manuscripta Math. 127 (2008), 469-484.

[3] R. SCHNEIDER, Convex bodies: The Brunn-Minkowski theory, Encyclopedia of Mathematics and its Applications, 44. Cambridge University Press, Cambridge, 1993.

[4] S. L. PAN, H. P. XU, Stability of a reverse isoperimetric inequality, J. Math. Anal. Appl. 350 (2009), 348-353.

[5] H. Groemer, Geometric applications of Fourier series and spherical harmonics, Encyclopedia of Mathematics and its Applications, 61. Cambridge University Press, Cambridge, 1996. 